\title{
Observations of a subcritical switch-on shock
}

\author{
H. Q. Feng ${ }^{1,2}$, J. M. Wang ${ }^{1}$, and J. K. Chao ${ }^{3}$ \\ 1 College of Physics and Electronic Information, Luoyang Normal University, Luoyang 471022, PR China \\ e-mail: hengqiangfeng@gmail.com \\ 2 State Key Laboratory of Space Weather, Center for Space Science and Applied Research, CAS, Beijing 100190, PR China \\ 3 Institute of Space Science, National Central University, Chungli 32001, Taiwan
}

Received 24 October 2008 / Accepted 19 May 2009

\section{ABSTRACT}

\begin{abstract}
Context. The conditions that lead to subcritical switch-on shocks are very rare in interplanetary space.
Aims. In this work, we report the identification of a subcritical switch-on shock driven by an interplanetary coronal mass ejection. Methods. The subcritical switch-on shock is identified by fitting the Rankine-Hugoniot (R-H) conditions.

Results. It is found that the shock has the following characteristics. (1) Proton density, temperature, and total magnetic field strength all increase from the upstream region to the downstream region. (2) Observed parameters meet the R-H relations closely. (3) The fast-mode Mach number is greater than unity in the preshock state and less than unity in the postshock state. (4) The normal AlfvénMach number is almost equal to 1 in the postshock state, while the upstream tangential magnetic component is around zero but the downstream tangential magnetic component is not zero (it is "switched on").

Conclusions. Considering that the shock parameters satisfy all the properties of subcritical switch-on shocks, we conclude that the discontinuity is a subcritical switch-on shock.
\end{abstract}

Key words. shock waves - Sun: coronal mass ejections (CMEs) - Sun: solar wind

\section{Introduction}

According to the MHD wave theory, four linear MHD waves exist and are called the fast magnetosonic wave, the Alfvén wave, the slow magnetosonic wave, and the entropy wave. The entropy wave has zero velocity with respect to plasma. If we define state 1 as the state where the fluid velocity is superfast-magnetosonic, state 2 where the fluid velocity is sub-fastmagnetosonic and super-Alfvénic, state 3 where the fluid velocity is sub-Alfvénic and super-slow-magnetosonic, and state 4 where the fluid velocity is sub-slow-magnetosonic, the entropysatisfying jump relations then include $1 \rightarrow 2$ (Viz. the fluid velocity transfer state 1 to state 2 across the shock front), $1 \rightarrow 3,1 \rightarrow 4$, $2 \rightarrow 3,2 \rightarrow 4$, and $3 \rightarrow 4$ transitions. The $1 \rightarrow 2$ and $3 \rightarrow 4$ shocks are fast and slow shocks, respectively. The four other transitions are called intermediate shocks (Todd 1964, 1965, 1966; Wu 1990). In addition, if the upstream flow velocity is equal to the normal Alfvén speed $\left(V_{\mathrm{A}}=B /\left(\mu_{0} \rho\right)^{1 / 2}\right)$, the transverse component of the downstream magnetic field vanishes, producing a slow switch-off shock (Landau \& Lifshitz 1984). As a result, switchoff shocks are the limiting cases of $3 \rightarrow 4$ slow shocks and $2 \rightarrow 4$ intermediate shocks. Similarly, switch-on shocks, which are denoted by $1 \rightarrow 2=3$, are limiting cases of $1 \rightarrow 2$ fast shocks and $1 \rightarrow 3$ intermediate shocks (Todd 1965; Wu 1990). Where $2=3$, then $V_{\mathrm{n}}=V_{\mathrm{An}}$ in the downstream region, that is, the normal Alfvén -Mach number is equal to 1 in the postshock state. Where $V_{\mathrm{n}}$ is the component of the bulk velocity normal to the shock front, $V_{\text {An }}$ is the Alfvén speed based on the magnetic-field component normal to shock front.

Switch-on shocks are a particular case of fast-mode shocks that lie at the boundary of the evolutionary rectangle (see Fig. 1) and propagate along the magnetic field. In this case, the flow

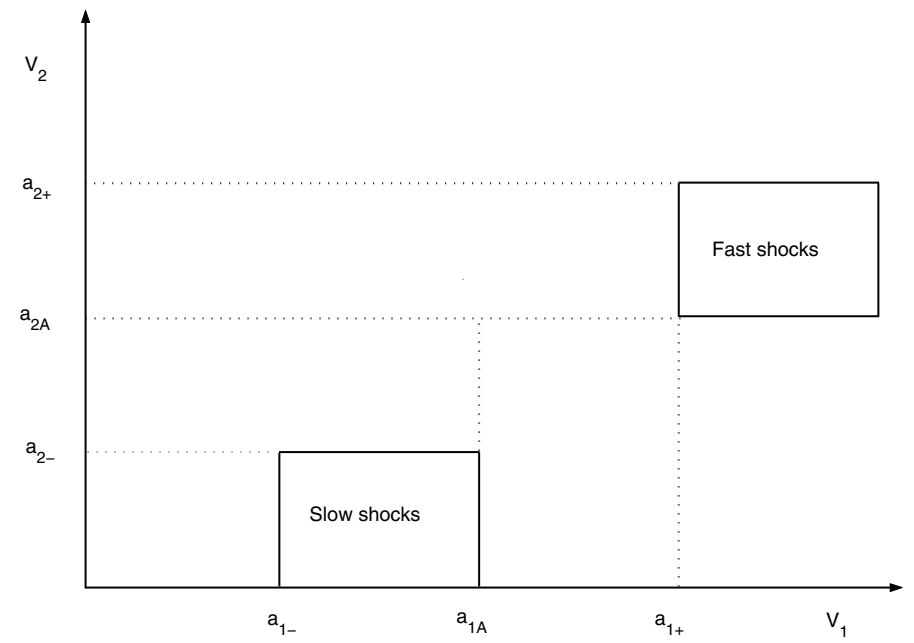

Fig. 1. The evolutionarity regions for the fast and slow shocks. Here $V_{i}$, $a_{i-}, a_{i A}, a_{i+}$ are velocities of the gas and of the small disturbances with respect to the jump before $(i=1)$ and after $(i=2)$ it (Barmin et al. 1996).

speed behind the shock is exactly equal to the intermediate speed (viz. Alfvén speed). The evolutionarity of switch-on shocks has been discussed by Akhiezer et al. (1958) and Syrovatskii (1959), where the ambient gas is treated as ideal and perfectly conducting. Thus the shock was treated as a discontinuity. They found that switch-on cannot emit enough small-amplitude plane waves to adjust themselves to small normal disturbances. Then theoretical study and numerical simulations showed that the switch-on shocks are admissible for cases in which the magnetic diffusivity 
of the ambient gas is much greater than any other diffusivity, which is a realistic situation (Barmin \& Gogosov 1960; Gogosov 1961; odd 1966; Landau \& Lifshitz 1984).

Switch-on shocks are evolutionary only until the Alfvén Mach number $M_{\mathrm{A}}$ becomes small enough that it is equal to a certain limiting value, $M_{\mathrm{A} *}=\sqrt{4-3 \frac{V_{\mathrm{S}}^{2}}{V_{\mathrm{A}}^{2}}}>1$, where $V_{\mathrm{S}}$ and $V_{\mathrm{A}}$ are the ordinary sound speed and the Alfvén speed, respectively (Kantrowitz \& Petschek 1966; Landau \& Lifshitz 1984). This means that switch-on shocks only occur in the low-plasma beta region. Therefore, if a fast CME moves in the low-beta solar wind, it may induce a switch-on shock (De Sterck et al. 1998). In addition, the complex bow-shock topology made switch-on shocks possible, and a switch-on shock has been observed in the Earth's bow shock (Farris et al. 1994). Switch-on shocks are easy to develop in the low plasma beta region; however, the plasma beta is high in interplanetary space, so switch-on shocks driven by CMEs are very rare in interplanetary space. Fortunately, we identify a subcritical switch on shock observed by Wind on October 31, 2001 by fitting the Rankine-Hugoniot ( $\mathrm{R}-\mathrm{H})$ relations. As stated previously, the rigid definition of a switch-on shock requires that the magnetic field and shock normal directions are aligned exactly, which is unrealistic on account of the effect of interplanetary perturbations. We therefore define subcritical switch-on shocks to be for which the angle, $\theta_{B N 1}$, between the shock normal and the upstream magnetic field vector is less than $15^{\circ}$. In this paper, we report the identification of a subcritical switch-on shock observed in interplanetary space.

\section{Observations of a subcritical switch-on shock}

\subsection{Data and method of analysis}

The magnetic field data obtained from magnetic field investigation (MFI) magnetometer and the proton data obtained from the 3-dimension plasma (3DP) analyzer are used for the subcritical switch-on shock identification. A description of the instruments onboard Wind can be found in Lepping et al. (1995) and Lin et al. (1995). The data have a time resolution of $3 \mathrm{~s}$. Here the coordinate system is the geocentric solar ecliptic (GSE) Cartesian system, where $x$ is along the Earth-Sun line and points to the Sun, $y$ points to the dusk in the ecliptic plane (opposing planetary motion), and $z$ points to the ecliptic north pole.

For an ideal MHD shock, the coplanarity theorem requires that the up- and down-stream magnetic fields $\left(\boldsymbol{B}_{1}\right.$ and $\left.\boldsymbol{B}_{2}\right)$ and the shock normal vector $\boldsymbol{n}$ lie on a coplanar plane. The shock normal vector can therefore be obtained as (Colburn \& Sonnet 1966)

$\boldsymbol{n}= \pm \frac{\left(\boldsymbol{B}_{1} \times \boldsymbol{B}_{2}\right) \times\left(\boldsymbol{B}_{1}-\boldsymbol{B}_{2}\right)}{\left|\left(\boldsymbol{B}_{1} \times \boldsymbol{B}_{2}\right) \times\left(\boldsymbol{B}_{1}-\boldsymbol{B}_{2}\right)\right|}$

so one can define an orthogonal shock coordinate system. Let $s$ denote the unit vector normal to the coplanar plane, which is calculated from $\boldsymbol{s}= \pm \frac{\left(\boldsymbol{B}_{1} \times \boldsymbol{B}_{2}\right)}{\left|\left(\boldsymbol{B}_{1} \times \boldsymbol{B}_{2}\right)\right|}$, then define $\boldsymbol{t}=\boldsymbol{n} \times \boldsymbol{s}$. Therefore, the $\boldsymbol{t}-\boldsymbol{s}$ plane is just the shock front, and both the up- and downstream magnetic fields are in the $\boldsymbol{n}-\boldsymbol{t}$ plane. To study an observed shock, it is important to fit measured magnetic fields and plasma on both sides to the $\mathrm{R}-\mathrm{H}$ relations. The main task of shock fitting is to set up an accurate shock frame of reference. In searching for an accurate shock frame, identification of the shock normal vector $\boldsymbol{n}$ is crucial. The shock normal can also be determined by the minimum variance analysis (MVA) method, which is based

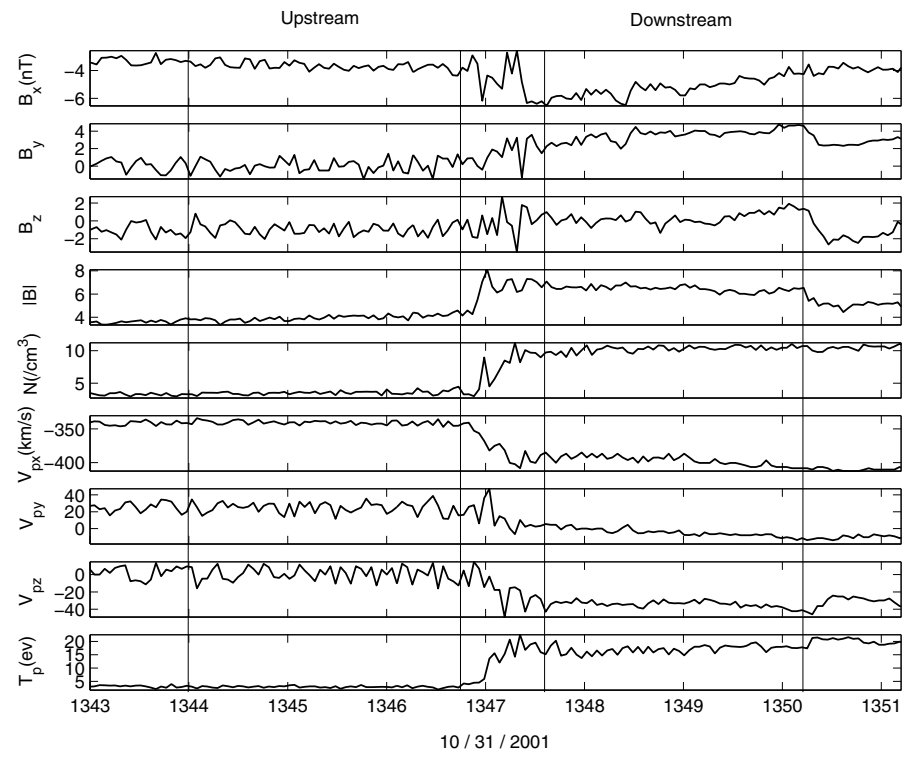

Fig. 2. The interplanetary magnetic field and plasma data measured by Wind in GSE coordinate system on October 31, 2001.

on the divergence free condition (Sonnerup \& Cahill 1967). But Knetter et al. (2004) suggest that the MVA method is much less reliable. Assuming that (1) a discontinuity surface can be approximated by a plane thin sheet (1D structure) and that (2) the speed of the discontinuity is constant in time and space, Knetter et al. (2004) used four spacecrafts (Cluster) to determine the discontinuity normal via triangulation.

In the present paper, we apply a new shock-fitting procedure proposed recently by Lin et al. (2006). Their method is based on one-fluid R-H relations, a Monte Carlo calculation, and a minimization technique. Lin et al. (2006) use the upstream and downstream observed mean variables and associated errors to randomly generate arrays of the variables needed for the fitting procedure, and all generated array, for these variables satisfy the $\mathrm{R}-\mathrm{H}$ relations. Here, each array is generated by using a randomnumber generator function, called $\operatorname{Rnd}(\sigma)$. This function generates an array of normally distributed numbers with an average of zero, and the standard deviation (SD) calculated from these numbers equals $\sigma$. Then a minimization technique are used, and with this, a best fit solution is obtained that satisfies the R-H relations and that is closest to the data mean. It should be noted that the total (associated) error consists of the SD and a systematic error that may be due to instruments and/or any other uncertainties. The SD can be calculated directly from the observable array. However, the systematic error is usually unknown; Lin et al. assumed that the systematic error is a half the SD. More detailed descriptions about the procedure can be found in Lin et al. (2006).

\subsection{Identification based on the $\mathrm{R}-\mathrm{H}$ relation}

This shock was observed at about 1347 UT on October 31, 2001, with Wind located at $(25.8,-83.8,7.0) R_{E}$ in the GSE coordinate system. About $8 \mathrm{~h}$ later, Wind observed an interplanetary coronal mass ejection (ICME), which can be found in the ICME list given by Cane \& Richardson (2003). The shock was driven by the ICME. Figure 2 shows the observed values of the parameters as functions of time for this event. From top to bottom, the panels show the $x, y, z$ components of the magnetic field $\left(B_{x}, B_{y}\right.$, $\left.B_{z}\right)$, the magnitude of the total magnetic field $(|B|)$, the proton 
Table 1. The observed and best-fitting parameters of the 2001 October 31 discontinuity event.

\begin{tabular}{lcc}
\hline \hline \multicolumn{1}{c}{ Parameter } & Observed values $^{a}$ & Best-fit values \\
\hline $\boldsymbol{B}_{1}(n T)$ & $(-3.65,-0.12,-1.09)$ & $(-3.91,-0.17,-1.57)$ \\
$\boldsymbol{B}_{2}$ & $(-4.58,3.73,0.40)$ & $(-4.48,3.300 .67)$ \\
$N_{1}, N_{2}\left(\mathrm{~cm}^{-3}\right)$ & $3.55,10.24$ & $3.75,10.04$ \\
$\boldsymbol{V}_{1}$ & $(-340.8,25.7,2.1)$ & $(-343.9,23.9,4.4)$ \\
$\boldsymbol{V}_{2}$ & $(-396.5,-4.1,-24.0)$ & $(-393.4,-2.3,-26.3)$ \\
$\beta_{1}, \beta_{2}$ & $0.657,2.852$ & $0.551,2.709$ \\
$\boldsymbol{n}$ & $(-0.943,-0.107,-0.316)$ & $(-0.947,0.050,-0.316)$ \\
$\boldsymbol{s}$ & $(-0.222,0.909,0.352)$ & $(-0.135,0.833,0.537)$ \\
$\boldsymbol{t}$ & $(0.250,0.402,-0.881)$ & $(0.290,0.551,-0.782)$ \\
$M_{\mathrm{AN} 1}, M_{\mathrm{AN} 2}$ & $1.836,1.080$ & $1.518,0.928$ \\
$M_{\mathrm{F} 1}, M_{\mathrm{F} 2}$ & $1.829,0.514$ & $1.509,0.529$ \\
$\theta_{\mathrm{BN} 1}$ & $4.82^{\circ}$ & $6.26^{\circ}$ \\
\hline
\end{tabular}

${ }^{a}$ The SD of $\boldsymbol{B}_{1}$ is $(0.30,0.66,0.62)$, the SD of $\boldsymbol{B}_{2}$ is $(0.66,0.74,0.64)$, the SD of $N_{1}$ and $N_{2}$ are 0.25 and 0.49 , the SD of $\boldsymbol{V}_{1}$ and $\boldsymbol{V}_{2}$ are (3.0, $5.8,7.6)$ and $(6.9,4.8,4.0)$, the $\mathrm{SD}$ of $\beta_{1}$, and $\beta_{2}$ are 0.087 and 0.117 , the error cone on the axes of the shock coordinate system is about $13^{\circ}$, the SD of $M_{\mathrm{AN} 1}, M_{\mathrm{AN} 2} 0.384$ and 0.169 , the SD of $M_{\mathrm{F} 1}, M_{\mathrm{F} 2}$ are 0.373 and 0.127 , the $\mathrm{SD}$ of $\theta_{\mathrm{BN} 1}$ is $6.42^{\circ}$.

density $(N)$, the $x, y, z$ components of the proton speed $\left(V_{\mathrm{p} x}\right.$, $\left.V_{\mathrm{p} y}, V_{\mathrm{p} z}\right)$, and temperatures $\left(T_{\mathrm{p}}\right)$. From Fig. 2 it can be seen that the proton number density $N$, the proton temperature $T_{\mathrm{p}}$, and the total magnetic field strength $|B|$ increase across the discontinuity. In addition, across the discontinuity, the proton velocity $|V|$ (not given in Fig. 2) also increased by $56 \mathrm{~km} \mathrm{~s}^{-1}$. All these jump signatures are consistent with the requirements for a fast shock.

The selected upstream regions are labeled by the two leftmost vertical lines in Fig. 2. Correspondingly, the regions between the two right most vertical lines are downstream. The selection of those intervals representative of the upstream and downstream regions is difficult and important, and a certain amount of subjectivity seems unavoidable. We try to select the intervals that are close to the transition layer when the magnetic field and plasma are relatively stable to reduce the effect of waves as well as the disturbances associated with the other structures. For this event, there are only coherent weak waveform fluctuates on both sides of the discontinuity, so it is easy to select the upstream and downstream regions.

The second column of Table 1 lists the observed averaged values of the magnetic filed vector $\boldsymbol{B}$, the solar wind velocity vector $\boldsymbol{V}$, the number density $N$, and the plasma beta $(\beta)$. Subscripts 1 and 2 refer to the upstream and downstream variables. We should note that Table 1 does not list the observed proton and electron temperatures, which are included in the $\beta$. Using the observed averaged values, we estimated the corresponding shock parameters. The derived parameters are the shock normal vector $\boldsymbol{n}$ (from Eq. (1)), the two other axes of the shock coordinate system $t$ and $s$, the normal Alfvén-Mach number $\left(M_{\mathrm{AN}}=V_{\mathrm{n}} / V_{\mathrm{An}}\right)$ in the upstream/downstream region, the fastmode Mach number $\left(M_{\mathrm{F}}=V_{\mathrm{n}} / V_{\mathrm{f}}\right)$ in the upstream/downstream region, and the shock normal angle, $\theta_{\mathrm{BN} 1}=\cos ^{-1}\left(\boldsymbol{B}_{1} \cdot \boldsymbol{n} / B_{1}\right)$, between the shock normal and the upstream magnetic field vector. In the above expression, $V_{\mathrm{An}}$ is the Alfvén speed based on the magnetic-field component normal to shock front $\left(V_{\mathrm{An}}=\right.$ $\left.B_{\mathrm{n}} /\left(\mu_{0} \rho\right)^{1 / 2}\right), V_{\mathrm{n}}$ is the component of the bulk velocity normal to the shock front and measured in the shock frame of reference, and $V_{\mathrm{f}}$ is the speed of the fast-magnetosonic wave in the direction of the shock normal, respectively.

Employing the fitting procedure of Lin et al. (2006), we obtain a best-fit solution, which satisfies the R-H relations.
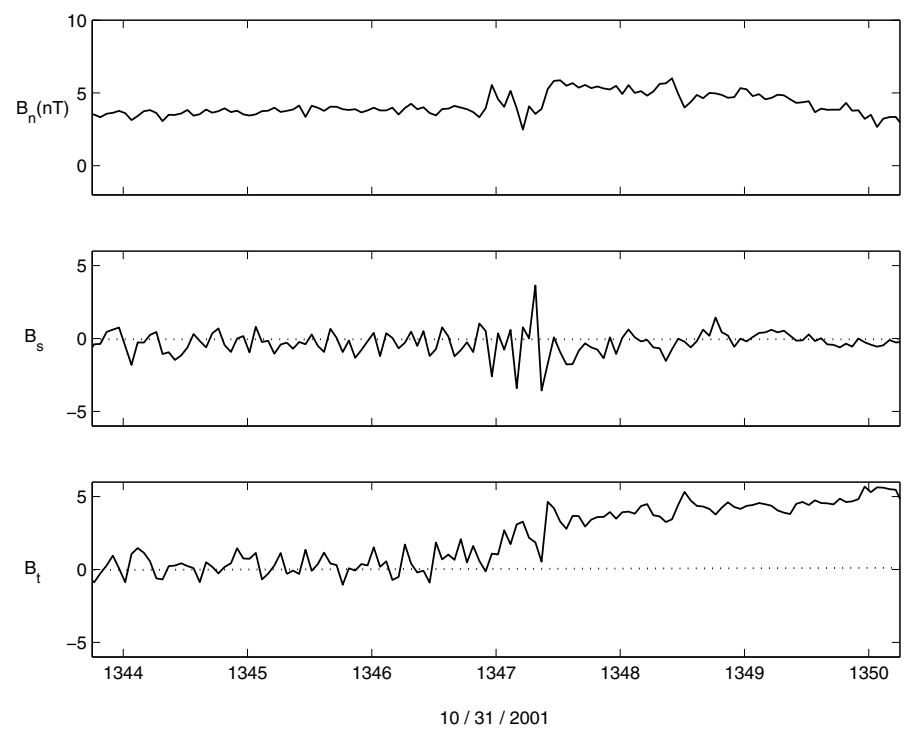

Fig. 3. The observed magnetic fields on 2001 October 31 in the shock coordinate system.

The third column of Table 1 lists the best-fit values and the corresponding parameters that are calculated from the fitting values. From Table 1, we found that this discontinuity has the following properties. (1) The fitting values are in strong agreement with the observed values, that is the observed parameters meet the R-H relations. (2) The proton density, temperature, and the total magnetic field strength increase from the upstream region to the downstream region. (3) The fast-mode Mach number is greater than unity in the preshock state and less than unity in the postshock state. (4) The normal Alfvén-Mach number $\left(M_{\mathrm{AN}}\right)$ is greater than unity in the preshock state and almost equal to unity in the postshock state (see Table 1$)$. (5) The angle $\left(\theta_{\mathrm{BN} 1}\right)$ between the shock normal and upstream magnetic field is very small. Figure 3 gives the magnetic-field profiles in the shock coordinate system. From Fig. 3, we can see that the upstream tangential magnetic field $B_{\mathrm{t}}$ is aligned almost along the shock normal; however, this component of the field becomes very large downstream of the shock ramp. The value of $B_{\mathrm{n}}$ approximately keeps constant, and the $B_{s}$ component is approximately zero. In addition, we also calculated the critical Alfvén Mach $M_{\mathrm{A} *}$. The calculated value is $M_{\mathrm{A} *}=1.535$, which is less than the estimated $M_{\mathrm{AN} 1}=1.836$ from observed mean variables. This conflict may be caused by the errors due to fluctuations of the observational data. However, the estimated $M_{\mathrm{AN} 1}=1.518$ from fitting variables is less than $M_{\mathrm{A} *}$, so we conclude that the discontinuity is a subcritical switch-on shock.

\section{Summary}

In more than four decades, there have been many investigations of interplanetary fast shocks. However, there are only a few of studies switch-on shocks. As Kennel \& Edmiston (1988) have pointed out, the switch-on shock is one of the peculiarities of MHD shock theory. At first sight, one would believe that a plane shock propagating parallel to the upstream magnetic field would be entirely hydrodynamic and would not change the magnetic field. On the other hand, below an upper limit normal AlfvénMach number to be defined, the hydrodynamic R-H relations lead to flow speeds that are less than the intermediate speed downstream, a violation of the MHD evolutionary conditions. The compromise effected by the full MHD R-H conditions is to 
"switch on" tangential components of magnetic field and flow velocity, so that the normal component of the flow speed precisely equals the intermediate speed downstream (Kennel \& Edmiston 1988). However, switch-on shocks are indeed evolutionary. They lie at the boundary of the corresponding evolutionary rectangle. As for the infrequent observations of switchon shock in interplanetary space, it may be that the plasma beta is high in interplanetary space. In this study, an interplanetary subcritical switch-on shock is identified by fitting the R-H relations. This subcritical switch-on shock was observed by Wind on October 31, 2001. The shock was driven by an ICME. We fitted this discontinuity using the procedure of Lin et al. (2006) and found that the observed magnetic field and plasma data satisfy the $\mathrm{R}-\mathrm{H}$ relations well. In addition, this shock has special characteristics of subcritical switch-on shocks: (1) the normal AlfvénMach number $\left(M_{\mathrm{AN}}\right)$ is greater than unity in the preshock state and almost equal to unity in the postshock state; (2) the upstream tangential magnetic component is around zero and the angle $\left(\theta_{\mathrm{BN} 1}\right)$ between the shock normal and upstream magnetic field is very small; however, downstream of the shock ramp, this component of the field becomes very large. We therefore believe that this event is a subcritical switch-on shock.

Acknowledgements. This work was supported by National Nature Science Foundation of China (NSFC) under grant Nos. 40804034, 10603014, 40890160. This work was also sponsored by Program for Science \& Technology Innovation Talents in Universities of Henan Province (HASTIT) under grant Nos. 2010HASTIT022, and by a Project Supported by the Specialized Research Fund for State Key Laboratories. The authors thank NASA/GSFC for the use of Wind MFI and 3DP obtained via the CDA Web page.

\section{References}

Akhiezer, A. I., Liubarskisl, S. I., \& Polovin, V. 1958, Soviet Phys. JETP., 8, 507 Barmin, A. A., \& Gogosov, V. V. 1960, Soviet Phys. Doklady, 134, 5, 1041

Barmin, A. A., Kulikovskiy, A. G., \& Pogorelsov, N. V. 1996, J. Comput. Phys., 126, 77

Cane, H. V., \& Richardson, I. G. 2003, J. Geophys. Res., 108, 1156

Colburn, D. S., \& Sonnet, C. P. 1966, Space Sci. Rev., 5, 439

De Sterck, H., \& Poedts, S. 1999, A\&A, 343, 641

De Sterck, H., Deconinck, H., Poedts, S., \& Roose, D. 1998, Int. Ser. num. math., 129,195

Daughton, W., Winske, D., \& Yin, L. 2001, Fully kinetic simulations of slowmode shocks (Berlin: Schaltungsdienst Lange o.H.G)

Farris, M. H., Russell, C. T., Fitzenreiter, R. J., \& Ogilvie, K. W., 1994, Geophys. Res. Lett., 21, 837

Gogosov, V. V. 1961, J. Appl. Math. Mech., 25, 141

Kantrowitz, A., \& Petschek, H. E. 1966, Plasma Physics in Theory and Application, (New York: McGraw-Hill), 147

Kennel, C. F., \& Edmiston, J. P. 1988 J. Geophys. Res., 93, 11363

Knetter, T., Neubauer, F. M., Horbury, T., \& Balogh, A. 2004, J. Geophys. Res., 109, A06102

Landau, L. D., \& Lifshitz, E. M. 1984, Electrodynamics of Continuous Media (Oxford: Pergamon Press)

Lepping, R. P., Acūna, M. H., Burlaga, L. F. et al. 1995, Space Sci. Rev., 71, 207 Lin, C. C., Chao, J. K., Lee, L. C., Lyu, L. H., \& Wu, D. J. 2006, J. Geophys. Res., 111, A09104

Lin, R. P., Anderson, K. A., Ashford, S. et al. 1995, Space Sci. Rev., 71, 125

Sonnerup, B. U, \& Cahill Jr, L. T. 1967, J. Geophys. Res., 72, 171

Syrovatskii, S. I. 1959, Sov. Phys., JETP. 8, 1024

Todd, L. 1964, J. Fluid Mech., 18, 321

Todd, L. 1965, J. Fluid Mech., 21, 193

Todd, L. 1966, J. Fluid Mech., 24, 597

Wu, C. C. 1990, J. Geophys. Res., 95, 8149 